\title{
Ameloblastic carcinoma of the maxilla: A case report and an updated review of the literature
}

\author{
ALESSANDRO MORO $^{1}$, ENRICO FORESTA ${ }^{1}$, GIULIO GASPARINI $^{1}$, SANDRO PELO $^{1}$, \\ MARIO FORCIONE $^{1}$, ENRICO GIUSEPPE CRISTALLINI ${ }^{2}$, MARCO TORALDO $^{2}$, \\ CARDARELLI LORENZO ${ }^{3}$, MARCO FALCHI $^{4}$ and GIANMARCO SAPONARO ${ }^{1}$ \\ ${ }^{1}$ Maxillofacial Surgery Unit, Faculty of Medicine, University Hospital 'A. Gemelli', \\ Catholic University of the Sacred Heart, I-00168 Rome; ${ }^{2}$ Pathological Anatomy Service, Foligno Hospital; \\ ${ }^{3}$ Private Practitioner; ${ }^{4}$ Department of Maxillofacial Surgery, Foligno Hospital, I-06034 Foligno, Italy
}

Received February 11, 2016; Accepted July 12, 2016

DOI: $10.3892 / \mathrm{ol} .2016 .5272$

\begin{abstract}
Ameloblastic carcinoma (AC) is an uncommon malignant odontogenic tumor that can be difficult to differentiate from ameloblastoma and can arise directly as an undifferentiated lesion or from a pre-existing benign lesion. The current study presents a novel case of primary maxillary $\mathrm{AC}$ and review the literature on $\mathrm{AC}$ of the maxilla. The review of the literature indicates that secondary tumors and posterior localization are associated with a higher tendency for recurrence and, often, multiple recurrences. Surgical therapy, eventually followed by radiotherapy, is the treatment modality most frequently applied, while the role of chemotherapy remains unclear. Several new cases of maxillary AC have been recently described in literature, making this pathology more frequent than previously considered; this is perhaps an indication of an increased diagnostic sensibility, rather than a real increase in the incidence of the disease itself.
\end{abstract}

\section{Introduction}

Malignant odontogenic tumors with the histological features of ameloblastoma represent $1.6-2.2 \%$ of all odontogenic tumors (1), and may be classified as metastasizing ameloblastoma or ameloblastic carcinoma (AC); it is generally agreed that the term 'malignant ameloblastoma' refers to tumors exhibiting features of a benign ameloblastoma, while the term 'ameloblastic carcinoma' indicates tumors that are still recognizable as ameloblastoma but possess histological features of malignancy. $\mathrm{AC}$, which seems to be more frequent than

Correspondence to: Dr Gianmarco Saponaro, Maxillofacial Surgery Unit, Faculty of Medicine, University Hospital 'A. Gemelli', Catholic University of the Sacred Heart, 3 Largo Agostino Gemelli, I-00168 Rome, Italy

E-mail: gianmarco.saponaro@gmail.com

Key words: ameloblastic carcinoma, ameloblastoma, malignant ameloblastoma, odontogenic tumor, maxillary tumor malignant ameloblastoma (2), is considered to be a rare tumor, as few cases have been published. Although rare, AC must be considered in the differential diagnosis of oral cavity tumors. In 2004 Adebiyi et al (2) conducted a clinicopathological analysis of 197 ectodermal odontogenic tumors and observed that $\mathrm{AC}$ was the most frequent odontogenic malignant tumor (5.6\%), followed by primary intraosseous carcinoma (1.5\%). $\mathrm{AC}$ exhibits a male predominance, may arise de novo or from a pre-existing odontogenic lesion (3), and affects a wide range of ages. Two-thirds of these tumors arise from the mandible, while one-third originate in the maxilla, and the posterior portion of the jaws is more commonly involved (4).

Since few cases have been reported in the literature, the incidence of the tumor, as well as the criteria for classification, are not precisely define. Furthermore, treatment modalities are still debated and there is lack of information regarding certain characteristics of the disease (5). In the present study, a clinical case of maxillary AC is presented and an up to date review of the published cases is done.

\section{Case report}

In August 2013, a 63-year-old female subject was referred to the Maxillofacial Surgery Unit of Foligno Hospital (Foligno, Italy) by her dentist, with a complaint of epistaxis and painful swelling in the left upper jaw (Fig. 1). The initial symptom was epistaxis, noted for the first time in February 2013; this was followed, in April 2013, by an ulcerated, rapidly growing and painful swelling localized in the upper maxillary fornix (Fig. 2). The patient was a non-smoker, reported no alcohol consumption, and had no contributing medical history. Extra-oral examination revealed facial asymmetry with swelling of the left cheek; no neurological abnormalities were noticed. Intra-oral examination revealed an ulcerated, swollen area in the posterior left upper jaw, involving the soft palate, the hard palate and the alveolar mucosa. Speech and feeding impairment were reported by the patient.

An incisional biopsy was performed under local anesthesia, and histological examination of paraffin-embedded tissue sections with $10 \%$ neutral embedded-buffered formalin established a provisional diagnosis of acanthomatous 
ameloblastoma. Immunostaining was performed with antibodies against cytokeratin 5 [monoclonal antibody specific for human cytokeratin 5 intermediate filament protein, used at pH 9.0 with Epitope Retrieval Solution 2 (Leica Biosystems Nussloch $\mathrm{GmbH}$, Nußloch, Germany) as antigen unmasker; Bond $^{\mathrm{TM}}$ Ready-to-Use Primary Antibody Cytokeratin 5; clone XM26; catalog no. PA0468; Leica Biosystems Nussloch $\mathrm{GmbH}$ ), cytokeratin 7 (mouse monoclonal antibody specific for human cytokeratin 7 intermediate filament, used at a dilution 1:50 at pH 6.0 with Epitope Retrieval Solution 2 as antigen unmasker; Novocastra ${ }^{\mathrm{TM}}$ Liquid Mouse Monoclonal Antibody Cytokeratin 7; clone OV-TL 12/30; catalog no. NCL-L-CK7-OVTL; Leica Biosystems Nussloch GmbH), p63 (monoclonal antibody used at pH 9.0 specific for human p63 protein, used with Epitope Retrieval Solution 2 as antigen unmasker; Bond ${ }^{\mathrm{TM}}$ Ready-to-Use Primary Antibody p63; clone: 7JUL; catalog no. PA0103; Leica Biosystems Nussloch GmbH), thyroid transcription factor-1 [TTF-1; mouse monoclonal antibody specific for human TTF-1, used at a dilution 1:200 at $\mathrm{pH} 6.0$ with Epitope Retrieval Solution 1 (Leica Biosystems Nussloch $\mathrm{GmbH}$ ) as antigen unmasker; Novocastra $^{\mathrm{TM}}$ Liquid Mouse Monoclonal, Antibody Thyroid Transcription Factor-1; clone SPT24; catalog no. NCL-L-TTF-1; Leica Biosystems Nussloch GmbH], cytokeratin 20 (mouse monoclonal antibody specific for human cytokeratin 20 intermediate filament protein, used at a dilution 1:50 at pH 6.0 with Epitope Retrieval Solution 1 as antigen unmasker; Novocastra ${ }^{\mathrm{TM}}$ Liquid Mouse Monoclonal Antibody Cytokeratin 20; clone Ks20.8; catalog no. NCL-L-CK20; Leica Biosystems Nussloch $\mathrm{GmbH}$ ), pan-cytokeratin (mouse monoclonal antibody specific for human cytokeratins 5,6,8 and 18 intermediate filament proteins, used at a dilution 1:80 with Enzyme 1 as antigen unmasker; Novocastra ${ }^{\mathrm{TM}}$ Lyophilized Mouse Monoclonal Antibody Cytokeratin (5/6/8/18); clones 5D3 and LP34; catalog no. NCL-CK5/6/8/18; Leica Biosystems Nussloch $\mathrm{GmbH}$ ) and $\mathrm{Ki}-67$ [mouse monoclonal antibody specific for human Ki-67 nuclear antigen (which is expressed in all proliferating cells during the G1, S, M and G2 phases of the cell cycle), used a dilution 1:100 at citrate $\mathrm{pH} 6.0$ as unmasking agent; Novocastra ${ }^{\mathrm{TM}}$ Liquid Mouse Monoclonal Antibody; clone MM1; catalog no. NCL-L-Ki67-MM-1;Leica Biosystems Nussloch $\mathrm{GmbH}]$.

A proliferation composed of solid epithelial cell nests with peripheral palisading was present. Neoplastic cells were focally atypical and scanty mitotic figures were evident. The Ki-67 proliferation index was $25 \%$. The presence of a high mitotic index and of focal nuclear atypia rendered necessary the study of further material to more accurately define the exact nature of the lesion. Computed tomography (CT) revealed an expansive lesion with erosion of the left maxillary sinus, with extension to the infratemporal fossa, nasopharynx, left nasal fossa, Eustachian tube and pterygoid muscles. The lesion was $\sim 58 \times 40 \mathrm{~mm}$, provoking left maxillary sinus expansion, and erosion of its medial and posterior wall, orbital floor and left pterygoid process (Fig. 3). Total body CT (TBCT) imaging revealed pathological bilateral cervical lymph nodes. Two parenchymal lesions were detected: One in the upper lobe of the left lung $(6 \mathrm{~mm})$, and one in the upper lobe of the right lung $(10 \mathrm{~mm})$. According to the histological results, the patient was diagnosed with an acanthomatous ameloblastoma

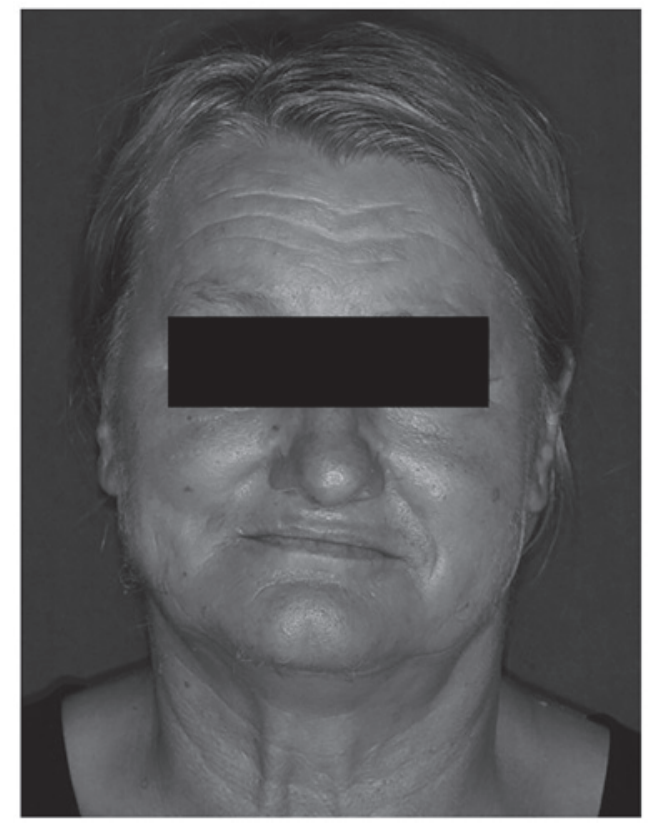

Figure 1. Extra-oral examination revealed facial asymmetry with swelling of the left cheek; no neurological abnormalities were noted.

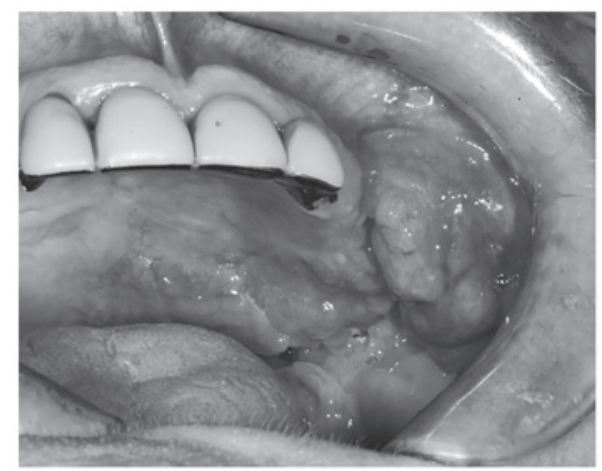

Figure 2. Intra-oral examination revealed an ulcerated swollen area in the posterior left upper jaw involving the soft palate, the hard palate and the alveolar mucosa.

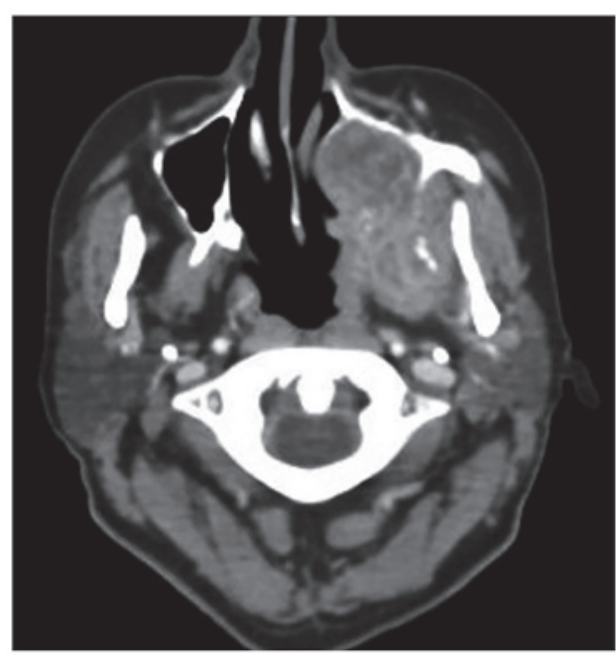

Figure 3. Computed tomography revealed small necrotic areas on the medial and posterior side of the reconstructive flap, extending to the tonsillar region, parapharyngeal space and masticatory space; a necrotic lymph node $(16 \mathrm{~mm}$ in diameter) was present in the right side (IIA area). 

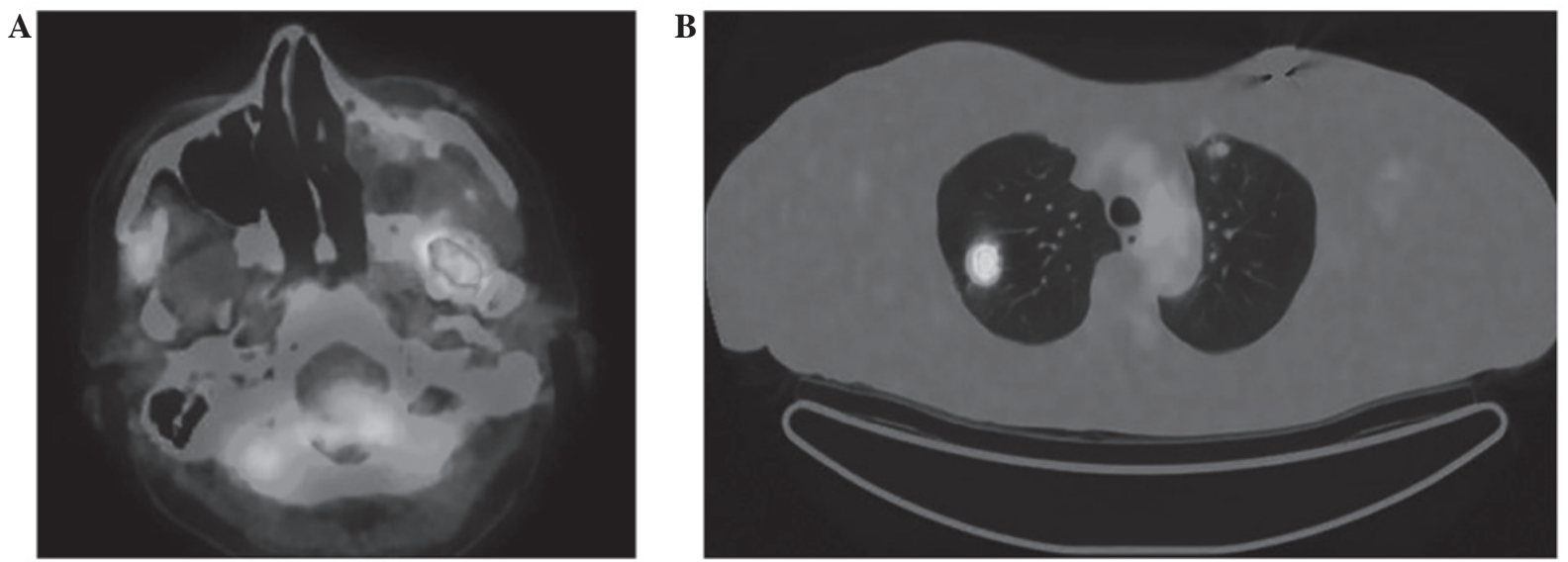

Figure 4. (A) Post-treatment F-fluorodeoxyglucose-positron emission tomography/computed tomography was performed to confirm the presence and the extension of recurrence, and (B) regional lymph node and distant metastases.

of the left maxilla. Due to the benign character of the lesion, the pathological cervical lymph nodes and the parenchymal lung lesions revealed on the TBCT could not be considered as secondary tumors.

The patient underwent a left posterior maxillectomy under general anaesthesia; the maxillectomy was extended to the infratemporal and pterygopalatine fossa. An immediate reconstruction was performed using a temporalis muscle flap. The histopathological diagnosis of the surgical specimen was AC; a proliferation made by an island of malignant epithelial cells embedded in a fibrous stroma was evident. The mass was partly solid and partly cystic. The basal cells of the islands appeared columnar, hyperchromatic and were aligned in a palisaded fashion; focally cell nuclei were displaced away from the basement membrane and their cytoplasm was vacuolated.

On immunohistochemistry, neoplastic cells were positive for cytokeratin 7 and negative for cytokeratin 20, and the Ki-67 proliferation index was $30 \%$. Following the surgical treatment, the patient was treated with 3 sessions of chemotherapy with cisplatin, 5-fluorouracil (5-FU) and cetuximab [1st session, $100 \mathrm{mg}$ cisplatin intravenous (IV) and $440 \mathrm{mg}$ cetuximab IV; 2nd and 3rd sessions, $150 \mathrm{mg}$ cisplatin IV, 6,000 mg 5-FU IV and $700 \mathrm{mg}$ cetuximab IV], followed by 35 sessions of radiotherapy (70 Gy, fractioned) to the primary site of the tumor and the neck. Head and neck CT was performed to evaluate the presence of local recurrence and the evolution of regional lymph nodes following therapies. CT revealed small necrotic areas on the medial and posterior side of the reconstructive flap, extending to the tonsillar region, parapharyngeal space and masticatory space; a necrotic lymph node was present in the right side (IIA area), measuring $16 \mathrm{~mm}$ in diameter.

F-fluorodeoxyglucose-positron emission tomography (FDG-PET)/CT was performed to confirm the presence and the extension of the recurrence and of regional lymph node and distant metastasis (Fig. 4). The FDG uptake confirmed the presence of local recurrence; the maximum standardized uptake value (SUVmax) was 10.4 on the left pterygoid muscle, 4.3 on the right superior deep jugular lymph node $(16 \mathrm{~mm})$, 8.6 on the right lung lesion and 3.8 on the left lung lesion. No uptake was documented on the left side of the neck. A core biopsy of the right, necrotic, jugular node was performed, confirming the presence of neck metastases of $\mathrm{AC}$, which were positive for Bcl-2, cytokeratin 7, cytokeratin 5 and p63, and negative for carcinoembryonic antigen. A right neck dissection and removal of the two lung nodes was performed, with histopathological findings indicating positive reactivity to cytokeratin 7, cytokeratin 5 and p63, and a diagnosis of AC metastases. The patient is currently undergoing radiotherapy following the removal of lung metastases. The patient signed an informed consent for publication of her pictures for scientific purposes.

\section{Discussion}

A literature review was conducted by searching the Pubmed (http://www.ncbi.nlm.nih.gov/pubmed), Scopus (https://www. scopus.com/) and Scholar (https://scholar.google.it/) databases using the keyword 'ameloblastic carcinoma'. Articles from 2014 to 2016 were selected among those published in English.

The statistics reported by Uzawa et al (6), which can be considered our starting point, have been updated, considering a total of 57 cases of maxillary AC (56 cases in literature plus the present case). A total of 14 cases not mentioned in previous reviews were identified: 13 from the literature review and 1 treated in our department (7-18). There were 2 cases reported in Uzawa's study [Krempien (1979), and Kruse and Zwahlen (2003)] (6) that were excluded as they were malignant ameloblastoma, which is not considered in the current review.

Of the reviewed patients, 44 were males and 13 were females, corresponding to a male:female ratio of 3.38:1. The mean age was 52.47, with a maximum of 90 and a minimum of 7 years. The most common symptoms leading to presentation were swelling $(66.66 \%)$, pain (25.49\%), ulcer (21.56\%) and bleeding $(9.8 \%)$.

A posterior location was reported in $61.40 \%$ (27 cases) of the cases, anterior location in $8.77 \%$ (4 cases), anterior and posterior location in $17.54 \%$ ( 8 cases), and the maxillary sinus was involved in $47.36 \%$ ( 21 cases) of the cases. In 7 patients, it was not determinable whether the tumor was primary or secondary; in the remaining 50 patients, 17 were secondary $(34.0 \%)$ and 33 were primary $(66.0 \%)$.

With regard to the primary treatment, surgical treatment was the modality of choice, and this was performed in 54 patients; in 35 patients, this was the only treatment 


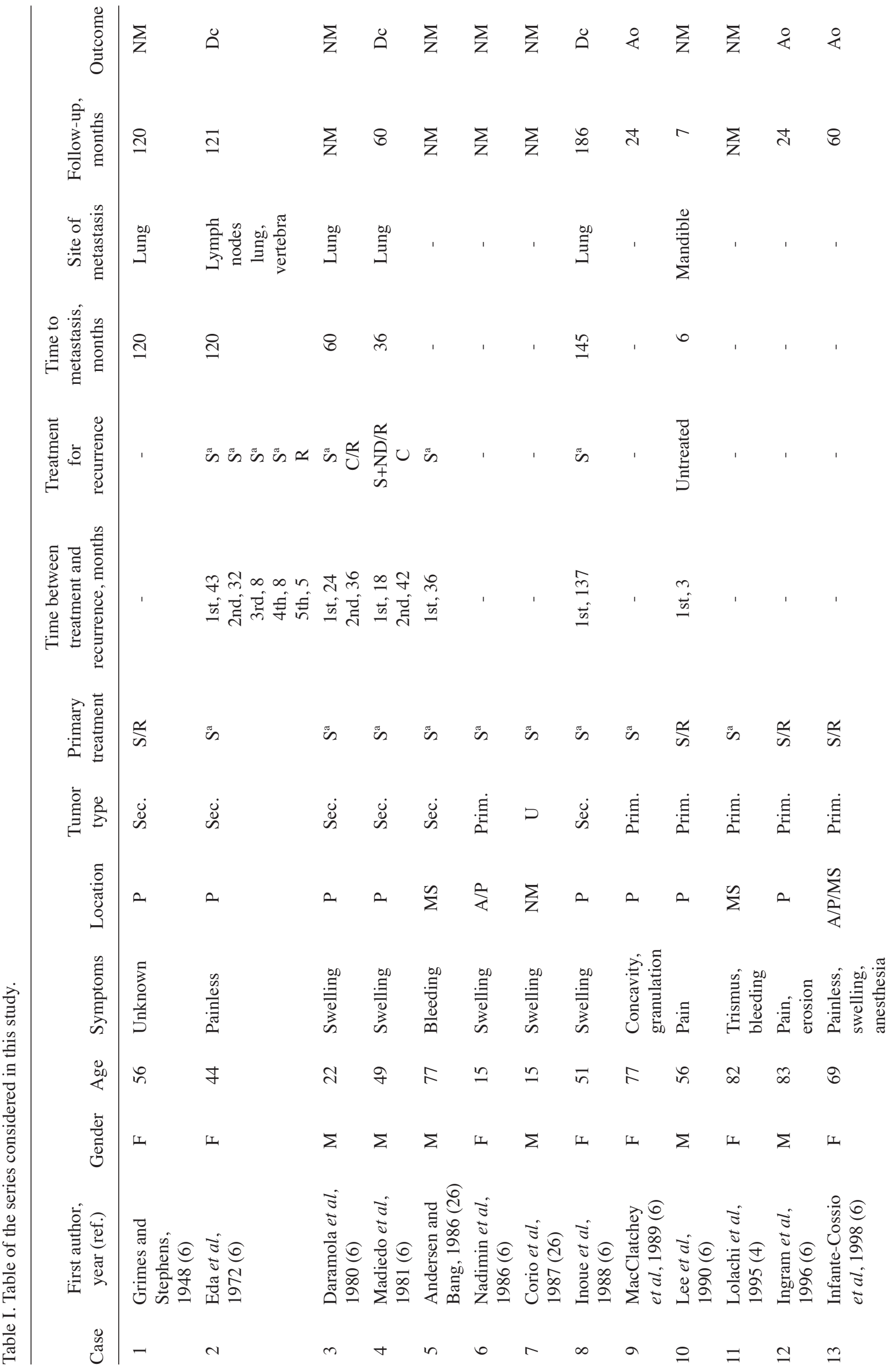


总

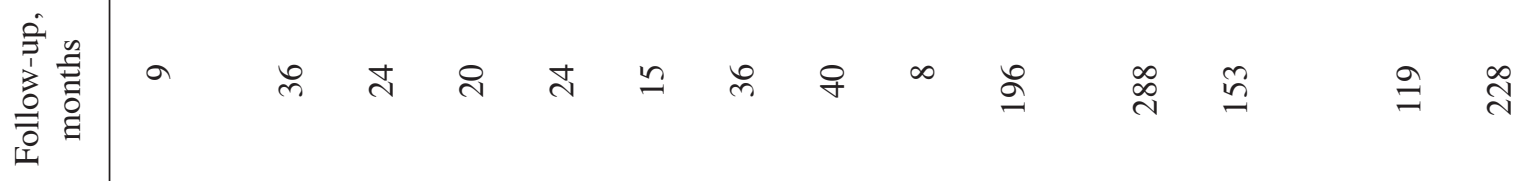

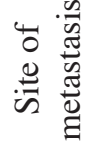

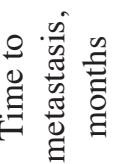

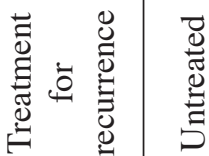

莺

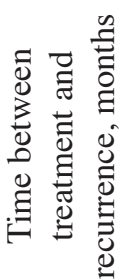

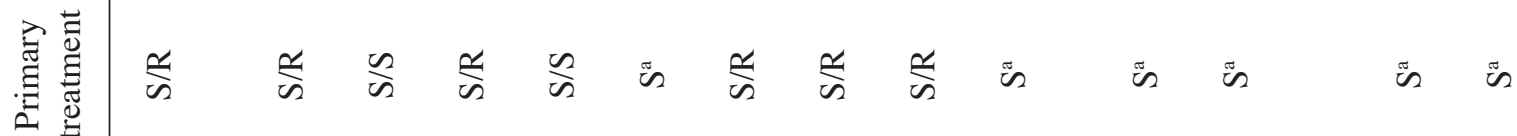

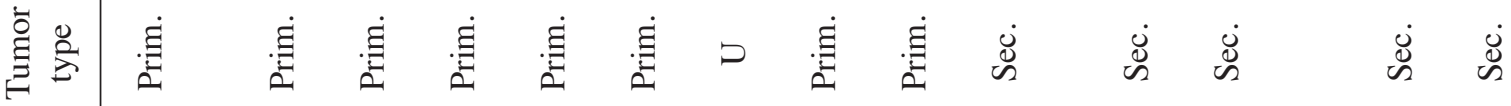

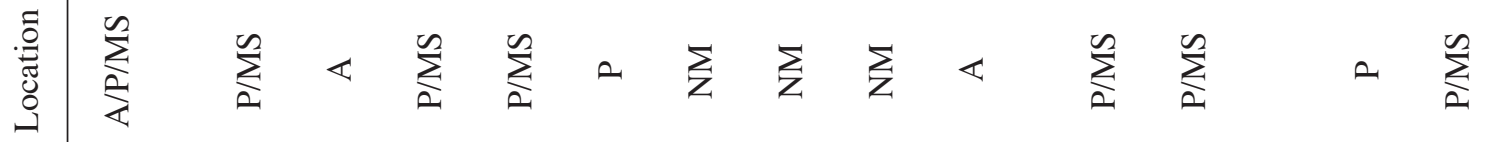

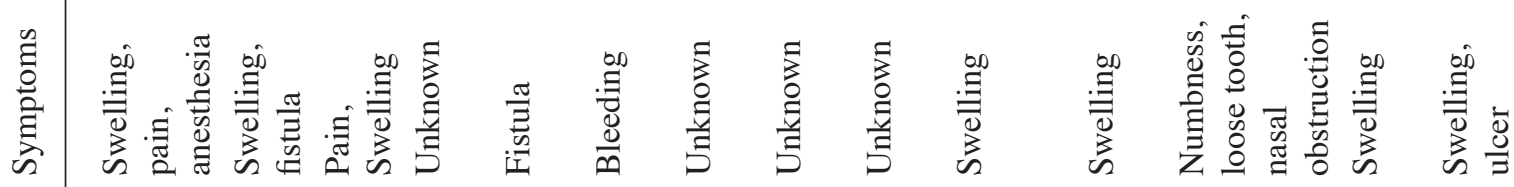

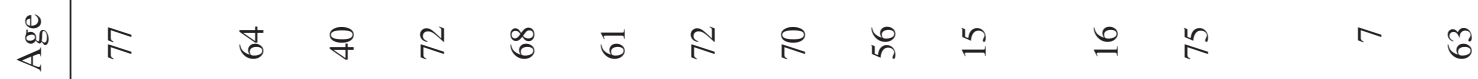

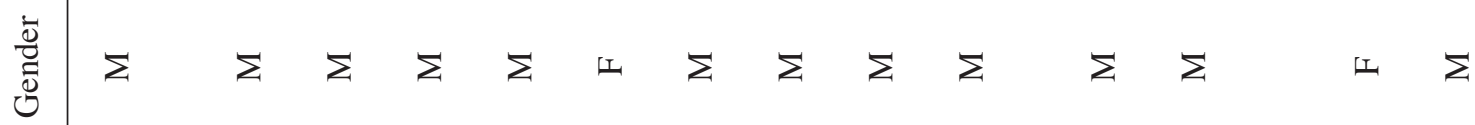

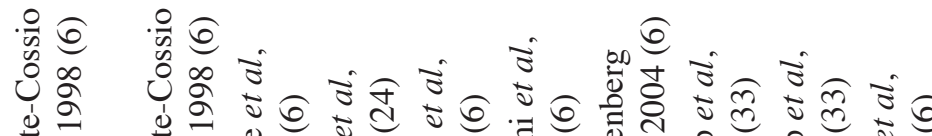

营灾

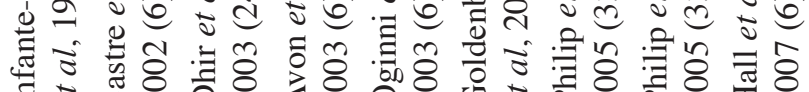

i $\overline{2} \approx$

ะ

蒂节

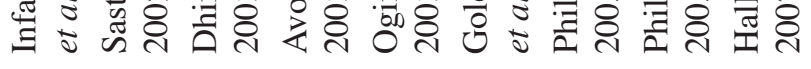

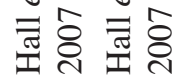

홒워

$\pm$

$\because \div=$

$=$

2

त)

สิ

व

ำ 


\begin{tabular}{|c|c|c|c|c|c|c|c|c|c|c|c|c|c|c|c|}
\hline $\begin{array}{l}\tilde{0} \\
0 \\
0\end{array}$ & 妄 & $\stackrel{2}{4}$ & $\stackrel{\circ}{ }$ & \& & \& & 是 & \& & $\frac{Q}{4}$ & \& & 安 & $\sum_{Z}$ & ڤే & $\ddot{~}$ & $\frac{Q}{4}$ & $\stackrel{0}{\&}$ \\
\hline 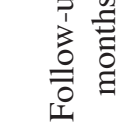 & $\vec{n}$ & F & $\stackrel{n}{a}$ & $\simeq$ & లి & ஃ & 0 & 0 & 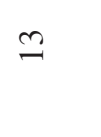 & $\vec{m}$ & $\sum_{Z}$ & $\simeq$ & $\nabla$ & $=$ & $\simeq$ \\
\hline 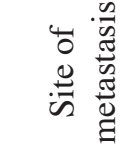 & $\stackrel{00}{\Xi} \stackrel{\vec{D}}{\Xi}$ & ' & ' & ' & ' & ' & ' & ' & ' & ' & ' & 1 & 1 & ' & ' \\
\hline 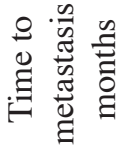 & F & 1 & ' & ' & 1 & ' & ' & ' & ' & ' & ' & ' & ' & ' & ' \\
\hline 节 & U & 1 & ' & ' & ' & ' & ' & ' & 敌 & ' & ' & 1 & ' & ' & ' \\
\hline 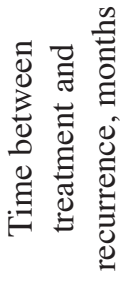 & 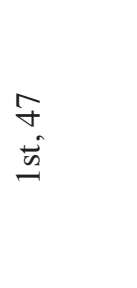 & ' & ' & ' & ' & ' & ' & ' & 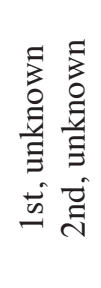 & ' & ' & 1 & 1 & ' & ' \\
\hline 窟泀 & שू & is & is & $\ddot{~[5 ~}$ & $\frac{\alpha}{n}$ & 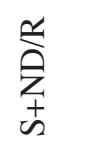 & $\frac{x}{\sqrt{n}}$ & in & $\frac{n}{n}$ & is & 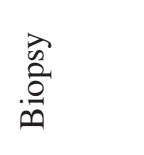 & 等 & 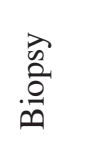 & is & ש̃ \\
\hline 寄 & $\begin{array}{l}\dot{j} \\
\tilde{n}\end{array}$ & $\dot{\Xi}$ & $\dot{\Xi}$ & $\dot{\Xi}$ & نू & $\dot{\Xi}$ & $\dot{\Xi}$ & 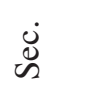 & $\dot{\Xi}$ & $\dot{\Xi}$ & $\dot{\Xi}$ & 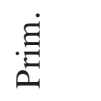 & $\dot{\Xi}$ & $\dot{\Xi}$ & $\dot{\Xi}$ \\
\hline 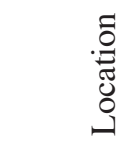 & $\sum_{\Sigma}^{\infty}$ & $\ll$ & $a$ & $\sum_{\sum}^{n}$ & $\sum_{a}^{\infty}$ & $\sum_{a}^{\infty}$ & $\sum_{i}^{\infty}$ & $\sum_{a}^{\infty}$ & $a$ & $\sum_{i}^{\infty}$ & $\sum_{a}^{\infty}$ & $a$ & $\sum_{i}^{\infty}$ & $a$ & $\sum_{\Omega}^{\infty}$ \\
\hline 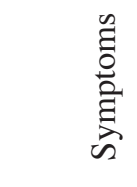 & 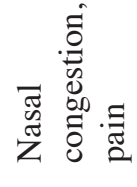 & 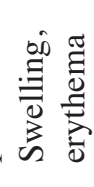 & 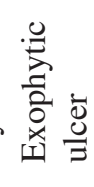 & $\begin{array}{l}\stackrel{\infty}{\Xi} \\
\overline{0} \\
\stackrel{0}{n}\end{array}$ & 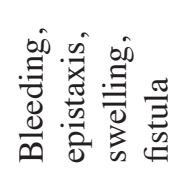 & 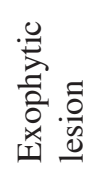 & 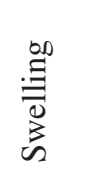 & 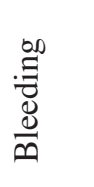 & 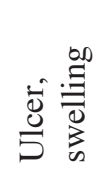 & 章离 & 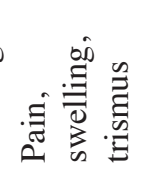 & 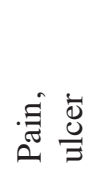 & $\begin{array}{l}\stackrel{\infty}{\Xi} \\
\stackrel{\Xi}{0} \\
\vdots \\
\infty\end{array}$ & 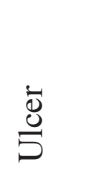 & $\begin{array}{l}\stackrel{\infty}{\Xi} \\
\overline{0} \\
\vdots \\
n\end{array}$ \\
\hline$\stackrel{8}{\infty}$ & in & $\vec{b}$ & ৪ & $?$ & $\check{a}$ & 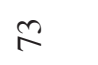 & 으 & $\infty$ & $\widehat{6}$ & $\Re$ & $\vec{b}$ & $\stackrel{\infty}{n}$ & $m$ & gิ & $\stackrel{m}{R}$ \\
\hline $\begin{array}{l}\overrightarrow{\bar{v}} \\
\overline{0} \\
\overline{0}\end{array}$ & $\Sigma$ & $\Sigma$ & $\Sigma$ & $\Sigma$ & $\Sigma$ & $\Sigma$ & $\Sigma$ & $\Sigma$ & $\Sigma$ & I & $\Sigma$ & $\Sigma$ & $\Sigma$ & $\Sigma$ & II \\
\hline 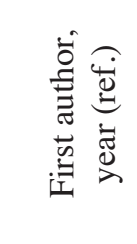 & 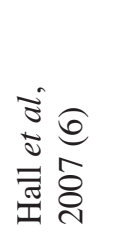 & 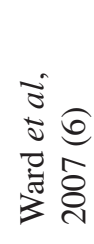 & 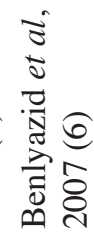 & 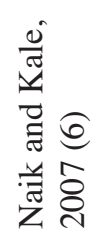 & 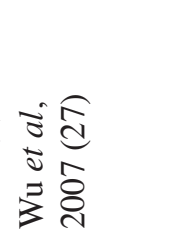 & 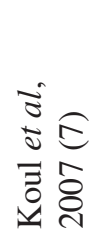 & 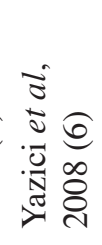 & 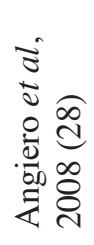 & 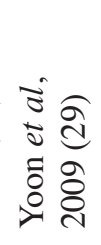 & 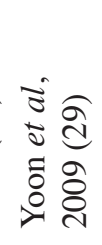 & 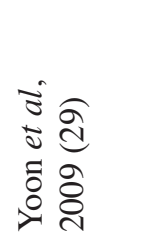 & 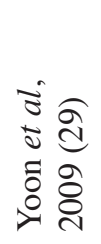 & 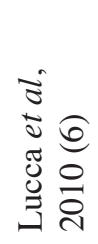 & 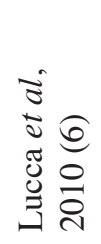 & 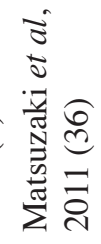 \\
\hline త్ & $\stackrel{\infty}{\sim}$ & ৯े & i & $\vec{m}$ & ก & $m$ & mे & $\ddot{n}$ & ల) & n & $\infty$ & ले & 우 & $\exists$ & ชั \\
\hline
\end{tabular}




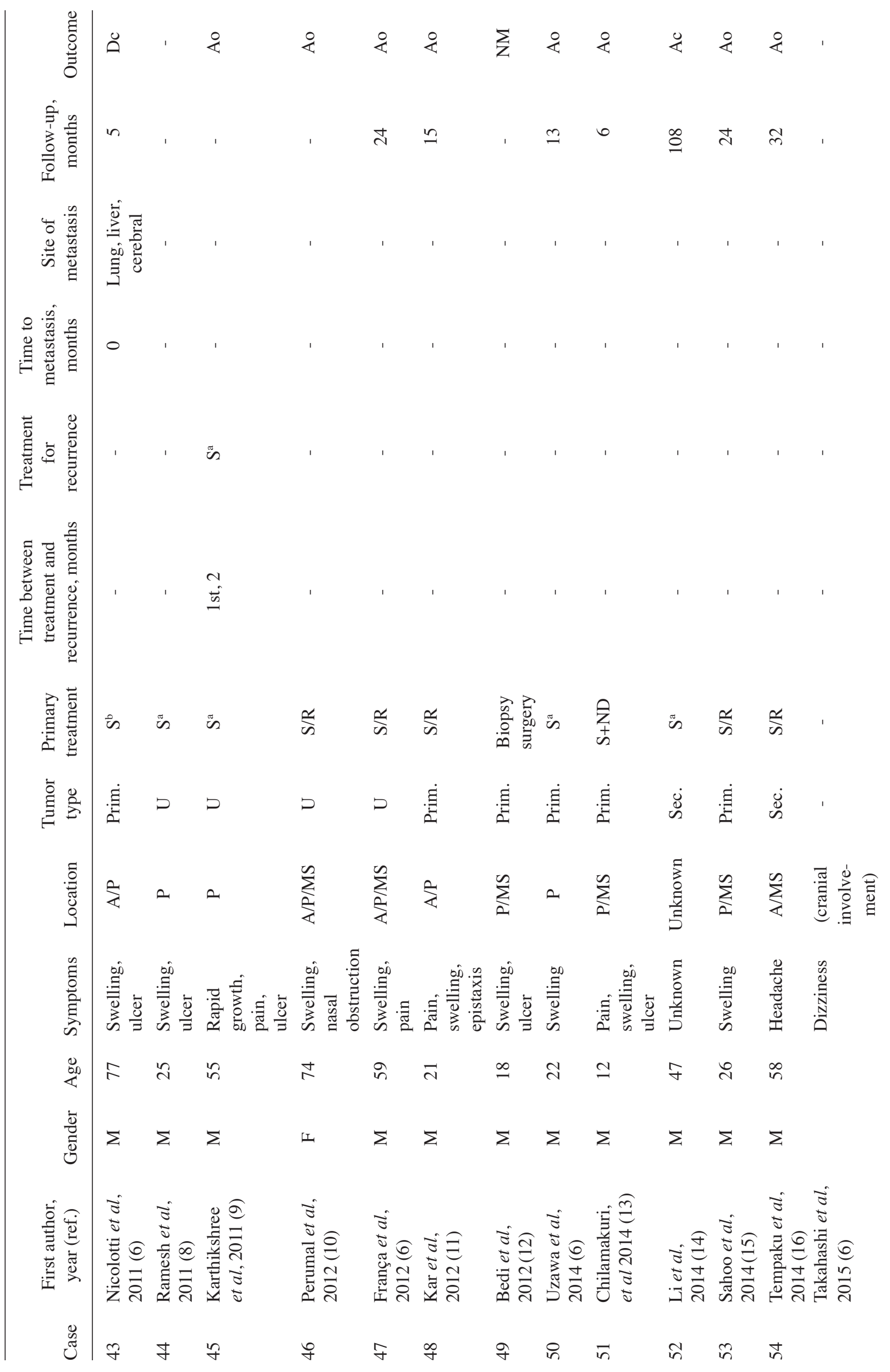




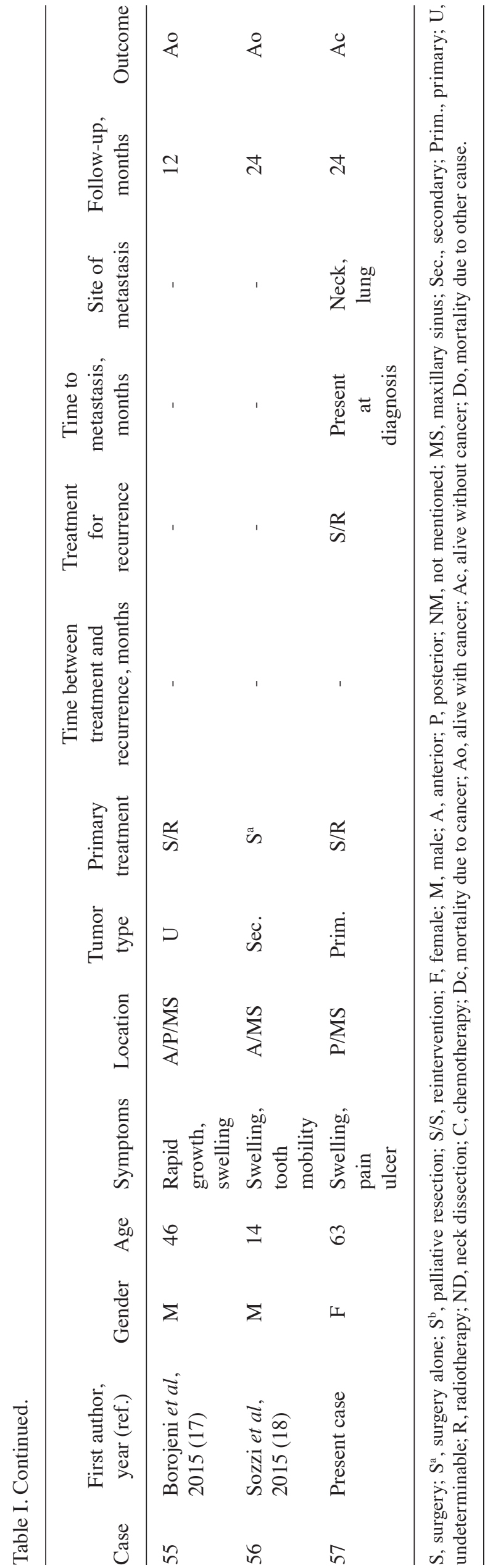

modality, while in 21 patients it was followed by radiotherapy, and in 3 cases a neck dissection was also performed.

Of the 57 total cases, 15 cases of recurrence were recorded (26.3\%): 11 recurrences $(73.3 \%)$ occurred in males, and 4 in females $(26,6 \%)$, with a male:female ratio of $2.75: 1$, an age range of 7-77 years and a mean age of 51.1 years. Of the recurrences, 12 occurred in cases with posteriorly located tumors $(80 \%)$, while 1 occurred in anterior, and 1 in anterior and posterior tumors; in 1 case, location was not mentioned. The predominance of posteriorly located tumors among recurrences was likely due to the high prevalence of posterior $(61.40 \%)$ or anteroposterior tumors (17.54\%) among all of the 57 included patients, compared to the lower prevalence of anterior maxillary $\mathrm{AC}(8.77 \%)$. Recurrences occurred predominantly in patients affected by 'secondary AC', and in 6 cases (40\%) multiple recurrences were observed (5 in secondary AC, 1 in primary AC). Of the cases of multiple recurrences, $83.33 \%$ occurred in patients with secondary AC. Additionally, 9 single recurrences were observed among the patients (5 in secondary, 3 in primary, and 1 in an unknown type of AC) (Table I).

In 1972, the World Health Organization (WHO) published a classification which mentioned malignant ameloblastoma (19). Subsequently, in 1982, Elzay (20) suggested a 'modification of the WHO classification system to include ameloblastic carcinoma' (Table II). In 1983, Shafer et al used the term 'ameloblastic carcinoma' to describe an ameloblastoma with features of malignancy in the primary or recurrent tumor (21). Other classifications have been proposed in order to distinguish $\mathrm{AC}$ from malignant ameloblastoma, a neoplasm in which the primary localization in the jaws shows features of a benign ameloblastoma, and which is typically recognized as 'malignant' once the metastases have been diagnosed. In 1984, Slootweg and Müller (22) noted that the diagnosis of malignant ameloblastoma was used in an indiscriminate way. For this reason the authors advocated a modification of the WHO classification in order to introduce a subgroup in which $\mathrm{AC}$ was described as arising de novo, ex ameloblastoma, or ex odontogenic cyst (Table III).

In the 2005 WHO classification (23), AC was defined as a rare odontogenic malignancy that combines the histological features of ameloblastoma with cytological atypia, even in the absence of metastases, and this is divided into primary, secondary intraosseous and secondary peripheral types (Table IV). The primary type arises as primitive malignancy of the jaws, and the secondary type is the malignant transformation of a pre-existing intraosseous or peripheral ameloblastoma. In 2009, Kruse et al (5) proposed a revision of the classification of AC, based on a review of the literature and with consideration of the origin and histopathological features of the primary tumor and metastases (Table V). Actually, the classifications of $\mathrm{AC}$ are still under revision, giving attention to histological features and biological behavior of the disease, which in the future may represent useful criteria for classification.

It is well known that $\mathrm{AC}$ is a disease that predominantly affects the adult population, with a predilection for males, and which is more frequent at the mandibular level rather than in the maxilla. Dhir et al (24) analyzed 17 cases of AC with a mean age of 53.5 years and male:female ratio of 1.5:1. Akrish et al (25) analyzed 37 cases of AC that were reported 
Table II. Elzay classification, 1982 (20).

\begin{tabular}{ll}
\hline Groups & \\
\hline Arising from an odontogenic cyst & Description \\
Arising from an ameloblastoma & \\
$2 \mathrm{a}$ & Well differentiated (malignant ameloblastoma) \\
$2 \mathrm{~b}$ & Poorly differentiated (ameloblastic carcinoma) \\
Arising de novo & \\
$3 \mathrm{a}$ & Non-keratinizing \\
$3 \mathrm{~b}$ & Keratinizing \\
\hline
\end{tabular}

Table III. Slootweg and Müller classification, 1984 (22).

\begin{tabular}{ll}
\hline Groups & \\
\hline Primary intraosseous carcinoma ex odontogenic cyst & Description \\
$2 \mathrm{a}$ & $\begin{array}{l}\text { Malignant ameloblastoma } \\
\text { 2b }\end{array}$ \\
& $\begin{array}{l}\text { Ameloblastic carcinoma, arising de novo, } \\
\text { ex ameloblastoma or ex odontogenic cyst }\end{array}$ \\
Primary intraosseous carcinoma de novo & Non-keratinizing \\
$3 \mathrm{a}$ & Keratinizing \\
\hline
\end{tabular}

Table IV. WHO classification, 2005 (23).

\begin{tabular}{ll}
\hline Type & Subgroups \\
\hline Ameloblastic carcinoma & Primary type \\
& Secondary type (dedifferentiated) intraosseous \\
& Secondary type (dedifferentiated), peripheral
\end{tabular}

between 1984 and 2007. In these patients, the male:female ratio was 2:1, the mean age was 52 years (range, 15-84 years), and the maxillary:mandibular tumor ratio was 1:1.9 (25). Li et al described a series of 12 patients treated at the West China Hospital of Stomatology between 2000 and 2008. The male:female ratio was 5:1 and the mean age was 44 years (range, 30-75 years). Mandibular localization was more frequent than maxillary tumors (ratio, 11:1), and there were 8 primary and 4 secondary tumors (14). In a review of the literature, Kar et al identified 92 cases reported between 1984 and 2012, with an age at presentation ranging from 7 to 91 years, a male:female ratio of 2.3:1, mandibular localization reported in 56 cases and maxillary localization in 35 cases (11). In a review on pediatric maxillary AC, Chilamakuri et al found a total of 104 cases of AC of the jaws reported between 1979 and August 2013; 18 cases were in children and adolescents, including 10 tumors located in the mandible and 8 in the maxilla. In the maxilla, these tumors occurred predominantly in the posterior region, and the age range of the patients was 5-18 years (13).

$\mathrm{AC}$ is considered a rare tumor, and maxillary $\mathrm{AC}$ is even less frequent; thus, knowledge of the disease is incomplete and general agreement regarding certain biological characteristics and the treatment of the disease has not been reached. Due to the lack of large clinical series and reports with long-term follow-up, several authors have recently reviewed data within the literature in order to collect information about the disease and verify the treatment options. In 2009, Kruse et al (5) presented a review of the literature on AC of the maxilla, including cases published between 1948 and 2008, identifying 26 cases; the patients had an average age of 54.4 years, with a marked prevalence in the group aged between 41 and 80 years (69.2\%), and a male:female ratio of 2.7:1. A similar review of the English literature published between 1948 and 2012, conducted by Uzawa et al (6), revealed 45 cases of maxillary $\mathrm{AC}$, with patients ranging in age between 5 and 90 years (mean age, 55.2 years) and a male:female ratio of 3:1 (6). Notably, following the review by Kruse, Uzawa found 19 new cases of maxillary AC that were published between 2008 and 2012, corresponding to an increase of $\sim 70 \%$ of reported cases over a period of 4 years. In the present review, the total number of cases of maxillary AC was 57, comprising patients with an average age of 52.4 years and a male:female ratio of 3.38:1.

The common clinical manifestations of AC are a large mass, which is often painless and rapidly growing, and which can be 
Table V. Kruse et al classification, 2009 (5).

Types of metastases

Description

Malignant ameloblastoma

a

b

Ameloblastic carcinoma arising from an ameloblastoma

a

b

c

Ameloblastic carcinoma with unknown origin histology (de novo)

a

b

c
Metastases with features of an ameloblastoma (well-differentiated) Metastases with malignant features (poorly differentiated)

Without metastases (malignant ameloblastoma)

Metastases with features of an ameloblastoma (well-differentiated)

Metastases with malignant features (poorly differentiated)

Without metastases

Metastases with features of an ameloblastoma (well-differentiated) Metastases with malignant features (poorly differentiated) associated with tooth mobility and bleeding if the oral mucosa is ulcerated. In maxillary AC, the predominant complaint is swelling, with gingival bleeding, trismus, dysphonia, paresthesia, epistaxis and oroantral fistulae also reported $(25,26,27)$. According to the current review, the most prevalent complaints were swelling (66.66\%), pain $(25.49 \%)$, and ulceration $(21.56 \%)$. At radiographic examination, a poorly defined radiolucency, often multilocular and with focal radiopacities, may be observed (19). Root resorption is also frequently observed (28); in cases of maxillary AC, this may be associated with sinus obliteration, palatal and maxillary bone resorption and nasal airway obstruction. A report by Angiero et al (28) highlighted that histological characterization of AC is challenging, and there are several differential diagnoses that must be excluded. The authors stated that the histological features of AC are like those of an ameloblastoma, showing foci with sheets, islands or trabeculae of epithelium, absence or rare presence of stellate reticulum, and round-to-spindle-shaped epithelial cells with little or no differentiation toward the columnar cells of ameloblastoma. Features that may be useful to differentiate AC from ameloblastoma are hyperchromatism, large or atypical nuclei, increased mitotic index, necrosis, calcification and, particularly, neural and vascular invasion. The presence of calcifications, which is not usual in ameloblastoma, and a proportion of clear cells $>15 \%$ suggest a diagnosis of AC. Angiero et al (28) also remarked that diagnosis can be difficult due to the variability of histological features, suggesting that aspects such as occasional mitoses, keratin production, and the formation of hyaline material near the epithelial portion of the tumor must be taken into consideration as indicative of malignant lesion (28).

In order to assist in differentiating AC from ameloblastoma, several immunohistochemical markers have been tested. Yoon et al (29) found that the expression of cytokeratin 18, parenchymal matrix metalloproteinase (MMP)-2, stromal MMP-9 and Ki-67 differentiate AC from ameloblastoma. Lei et al (30) recommend the inclusion of SOX2 immunohistochemical staining in conjunction with Ki-67 in challenging cases with atypical features. Locally the tumor is invasive, often showing a silent infiltrative growth (30).
In a review of 31 studies published between 2005 and 2011, Casaroto et al (31) found a high incidence of primary $\mathrm{AC}$ in the maxilla, while secondary $\mathrm{AC}$ was more frequent in the mandible. In the review, secondary AC was associated with cases with recurrence or mortality from the disease. The higher frequency of the primary type in the maxilla and the more aggressive behavior of the secondary type are confirmed by other studies $(32,33)$. In the present series of 57 maxillary AC, the tumor types of 7 cases were not determined due to a lack of available information. In the remaining 50 cases, there were 17 secondary (34\%) and 33 primary tumors $(66 \%)$.

A general consensus on treatment modalities for AC has still not been reached; however, surgical therapy is the most widely used treatment option, while few data regarding chemotherapy are available (34). The recurrence rate following curettage of maxillary ameloblastoma is almost $100 \%$ (25); Yoon et al (29) reported a recurrence rate of $92.3 \%$ following curettage alone and $28.3 \%$ following partial resection, and therefore a wide surgical resection, with clear margins free of tumor, is recommended $(5,28,34)$. Taking into consideration the relatively lower malignancy of primary $\mathrm{AC}$, a more tissue sparing resection may be indicated for primary tumors, while a wider resection is recommended in cases of secondary $\mathrm{AC}(6,28)$. Radiotherapy is used in cases with positive surgical margins, perineural infiltration or soft-tissue invasion (33).

Stereotactic radiosurgery (SRS) in the treatment of $\mathrm{AC}$ has been recently reported (34). Takahashi et al (35) presented a report of the efficacy of single-fraction helical tomotherapy for the treatment of residual $\mathrm{AC}$ following surgical resection, remarking that SRS may be an effective treatment, but only for small volumes due to the high doses used in radiosurgery.

$\mathrm{AC}$ is prone to metastasis, and the presence of hematogenous spread (the most frequent mode of diffusion) or neck node involvement must be verified. In the review by Uzawa et al (6), regional metastases occurred in 3 cases: 1 in the mandible and 2 in the lymph nodes of the neck. The most common location of distant metastasis was the lung, occurring in 9 cases. Distant metastases were also reported in the liver in 2 cases, 
the bone in 2 cases, the brain in 1 case and the myocardium in 1 case. In the review by Kruse et al (5), pulmonary metastases were identified in $34.6 \%$ of cases, and cervical lymph node metastasis could be found in only 1 patient (5). The present review of the existing literature found 2 cases of neck node involvement and 9 of distant metastases, while the current case treated at our hospital exhibited neck node involvement and lung metastases at the time of diagnosis. Indications for neck dissection remain unclear; however, the procedure is not recommended if there is no evidence of lymph node involvement $(28,29)$.

Recurrence of AC is not infrequent and appears to be associated with mortality. Yoon et al (29) reported a recurrence rate of $28.3 \%$ in patients who underwent surgical resection, while Uzawa et al (6) found a rate of recurrence of $38.4 \%$, often involving multiple recurrences or associated with metastases (6). The present review identified 15 recurrences among 57 cases $(26.6 \%)$; the majority of recurrences were in cases of posterior tumor location ( $80 \%)$. This is likely due to the higher prevalence of posterior $(61.40 \%)$ or anteroposterior $(17.54 \%)$ tumors, compared to the low prevalence of anterior maxillary $\mathrm{AC}(8.77 \%)$, in this review.

Infiltration of surgical margins, presence of metastases and diagnostic delay are other factors associated with poor prognosis (25). Uzawa et al (6) recommended early, aggressive and complete removal of the tumor as the best treatment to improve the survival rate, and a more radical treatment modality in cases with primary recurrence. For the same reason an early and precise diagnosis is mandatory, and it has been suggested that FDG-PET/CT should be routinely performed during the diagnostic phase in order to reveal possible metastatic disease $(6,36)$ or, together with magnetic resonance imaging, to detect tumor margins and plan surgical resection (36). Postoperatively, ${ }^{11} \mathrm{C}$-methionine (MET)-PET can be useful in order to detect residual disease or recurrence during follow-up (27). Since lesional MET accumulation has been observed to decrease concurrently with the decreased tumor volume following treatment with single-fraction radiotherapy, MET-PET may also be used in monitoring the response of AC to radiotherapy (27).

As previously reported, the prognosis of AC is heavily influenced by the persistence of the tumor, the presence of metastases and recurrences. Primary AC shows a more favorable prognosis than secondary $\mathrm{AC}$, and young patients have a higher survival rate compared with the older patients (27). In any case, lifetime follow-up is recommended due to the possibility of late recurrence of the tumor $(5,6)$.

In summary, $\mathrm{AC}$ is considered a rare disease and the present review of the literature demonstrates the lack of knowledge regarding this tumor and the consequent ongoing debate regarding certain aspects of this disease. In recent years, a substantial number of new cases of AC have been published and the disease has been investigated with greater attention. It is possible that these new cases should be attributed to the higher level of awareness of the disease and increased accuracy of diagnoses, rather than to a true increase in the incidence of AC. If this is the case, this may indicate that better knowledge of the disease accounts for an increased diagnostic sensibility, and, thus, AC may not be as rare as previously believed.

\section{References}

1. Rizzitelli A, Smoll NR, Chae MP, Rozen WM and Hunter-Smith DJ: Incidence and overall survival of malignant ameloblastoma. PLoS One 10: e0117789, 2015.

2. Adebiyi KE, Odukoya O and Taiwo EO: Ectodermal Odontogenic tumours: Analysis of 197 Nigerian cases. Int J Oral Maxillofac Surg 33: 766-770, 2004

3. Cizmecý O, Aslan A, Onel D and Demiryont M: Ameloblastic carcinoma ex ameloblastoma of the mandible: Case report. Otolaryngol Head Neck Surg 130: 633-634, 2004.

4. Lolachi CM, Madan SK and Jacobs JR: Ameloblastic carcinoma of the maxilla. J Laryngol Otol 109: 1019-1022, 1995.

5. Kruse AL, Zwahlen RA and Grätz KW: New classification of maxillary ameloblastic carcinoma based on an evidence-based literature review over the last 60 years. Head Neck Oncol 1: 31, 2009.

6. Uzawa N, Suzuki M, Miura C, Tomomatsu N, Izumo T and Harada K: Primary ameloblastic carcinoma of the maxilla: A case report and literature review. Oncol Lett 9: 459-467, 2015.

7. Koul R, Binahmed A, Dubey A, Nason R and Cooke AL: Maxillary Ameloblastic Carcinoma. J HK Coll Radiol 11: 32-34, 2008.

8. Ramesh M, Sekar B, Murali S, Mathew S, Chacko J and Paul G: Ameloblastic carcinoma - review and histopathology of 5 cases. Oral Maxillofac Pathol J (OMPJ) 2: 154-160, 2011.

9. Karthikshree V, Prashad Ramesh V, Balamurali PD and Premalatha B: Ameloblastic Carcinoma - A case report highlighting its variations in histology. J Int Oral Health 3: 37-42, 2011.

10. Perumal CJ: Ameloblastic carcinoma of the maxilla with extension into the ethmoidal air cells and close proximity to the anterior skull base: A rare case presentation. Craniomaxillofac Trauma Reconstr 5: 169-174, 2012.

11. Kar IB, Subramanyam RV, Mishra N and Singh AK: Ameloblastic carcinoma: A clinicopathologic dilemma - Report of two cases with total review of literature from 1984 to 2012. Ann Maxillofac Surg 4: 70-77, 2014.

12. Bedi RS, Chugh A and Pasricha N: Ameloblastic carcinoma of maxilla. Natl J Maxillofac Surg 3: 70-74, 2012.

13. Chilamakuri S, Murathati S, Nuvvula S, Nirmala S, Devireddy SK and Kanubaddy SR: Pediatric maxillary ameloblastic carcinoma. J Cranio Max Dis 3: 141-146, 2014.

14. Li J, Du H, Li P, Zhang J, Tian W and Tang W: Ameloblastic carcinoma: An analysis of 12 cases with a review of the literature. Oncol Lett 8: 914-920, 2014.

15. Sahoo TK, Samal S, Dhal I, Mishra C and Kar A: Ameloblastic carcinoma of maxilla and its management: A case report and review of literature. Int J Dent Med Res 1: 73-76, 2014.

16. Tempaku A, Takahashi Y, Ikeda H, Yamauchi S, Gotoh T, Bandoh N, Makino S, Shimada T and Kamada H: Usefulness of 11C-methionine positron emission tomography for detecting intracranial ameloblastic carcinoma: A case report. Oncol Lett 8: 1509-1512, 2014.

17. Borojeni MP and Etesami M: Ameloblastic carcinoma of maxillary sinus. Int J Dent Med Res 1: 73-75, 2015.

18. Sozzi D, Morganti V, Valente GM, Moltrasio F, Bozzetti A and Angiero F: Ameloblastic carcinoma in a young patient. Oral Surg Oral Med Oral Pathol Oral Radiol 117: e396-e402, 2014.

19. Pindborg JJ, Kramer IRH and Torloni H (eds): Histological typing of odontogenic tumors, jaw cysts and allied lesions. World Health Organisation, Geneva, pp35-36, 1972.

20. Elzay RP: Primary intraosseous carcinoma of the jaws. Review and update of odontogenic carcinomas. Oral Surg Oral Med Oral Pathol 54: 299-303, 1982.

21. Shafer WG, Hine MK and Levy BM (eds): A Textbook of Oral Pathology. 4th edition. WB Saunders, USA, pp251-258, 1983.

22. Slootweg PJ and Müller H: Malignant ameloblastoma or ameloblastic carcinoma. Oral Surg Oral Med Oral Pathol 57: 168-176, 1984.

23. Barnes L, Eveson JW, Reichart P and Sidransky D (eds): World Health Organization Classification Of Tumours - Pathology \& Genetics: Head and Neck Tumours. IARC Press, Lyon, France, 2005.

24. Dhir K, Sciubba J and Tufano RP: Ameloblastic carcinoma of the maxilla. Oral Oncol 39: 736-741, 2003.

25. Akrish S, Buchner A, Shoshani Y, Vered M and Dayan D: Ameloblastic carcinoma: Report of a new case, literature review, and comparison to ameloblastoma. J Oral Maxillofac Surg 65: 777-783, 2007. 
26. Corio RL, Goldblatt LI, Edwards PA and Hartman KS: Ameloblastic carcinoma: a clinicopathologic study and assessment of eight cases. Oral Surg Oral Med Oral Pathol 64: 570-576, 1987.

27. Wu JY, Chi LH, Pemg BY and Lin YH: Ameloblastic carcinoma of the maxilla - case report. J Dent Sci 2: 164-170, 2007.

28. Angiero F, Borloni R, Macchi M and Stefani M: Ameloblastic carcinoma of the maxillary sinus. Anticancer Res 28: 3847-3854, 2008.

29. Yoon HJ, Hong SP, Lee JI, Lee SS and Hong SD: Ameloblastic carcinoma: An analysis of 6 cases with review of the literature. Oral Surg Oral Med Oral Pathol Oral Radiol Endod 108: 904-913, 2009

30. Lei Y, Jaradat JM, Owosho A, Adebiyi KE, Lybrand KS Neville BW, Müller S and Bilodeau EA: Evaluation of SOX2 as a potential marker for ameloblastic carcinoma. Oral Surg Oral Med Oral Pathol Oral Radiol 117: 608-616.e1, 2014.

31. Casaroto AR, Toledo GL, Filho JL, Soares CT, Capelari MM and Lara VS: Ameloblastic carcinoma, primary type: Case report, immunohistochemical analysis and literature review. Anticancer Res 32: 1515-1525, 2012.
32. Henderson JM, Sonnet JR, Schlesinger C and Ord RA: Pulmonary metastasis of ameloblastoma: Case report and review of the literature. Oral Surg Oral Med Oral Pathol Oral Radiol Endod 88: 170-176, 1999

33. Philip M, Morris CG, Werning JW and Mendenhall WM: Radiotherapy in the Treatment of Ameloblastoma and Ameloblastic Carcinoma. J HK Coll Radiol 8: 157-161, 2005.

34. Perera E, Lindquist C, Hughes $\mathrm{C}$ and Thomas S: The use of gamma knife stereotactic radiosurgery in the treatment of ameloblastic carcinoma. Int J Oral Maxillofac Surg 42: 934-938, 2013

35. Takahashi $\mathrm{Y}$, Bandoh N, Miyamoto $\mathrm{A}$ and Kamada H: Single-fraction helical tomotherapy for ameloblastic carcinoma. J Oral Maxillofac Surg 74: 302-306, 2016.

36. Matsuzaki H, Katase N, Hara M, Asaumi J, Yanagi Y, Unetsubo T, Hisatomi M, Konouchi $\mathrm{H}$ and Nagatsuka H: Ameloblastic carcinoma: A case report with radiological features of computed tomography and magnetic resonance imaging and positron emission tomography. Oral Surg Oral Med Oral Patho Oral Radiol Endod 112: e40-e47, 2011. 